\title{
toolbox
}

\section{The Centres for Health Evidence Website (www.cche.net)}

Derek Richards

Director, Centre for Evidence-based Dentistry

The Centres for Health Excellence (CHE) is a Canadian not-for-profit organisation funded by grants and service contracts. The $\mathrm{CHE}$ is involved in projects and partnerships that promote practice-based evidence. The overall goal of the $\mathrm{CHE}$ is to promote evidence-based health care by presenting knowledge-based resources to health professionals in ways that facilitate their optimum use.

Evidence-Based Dentistry (2002) 3, 56-59. doi:10.1038/sj.ebd.6400109

The CHE has a number of objectives under the headings infostructure, instruction and investigation.

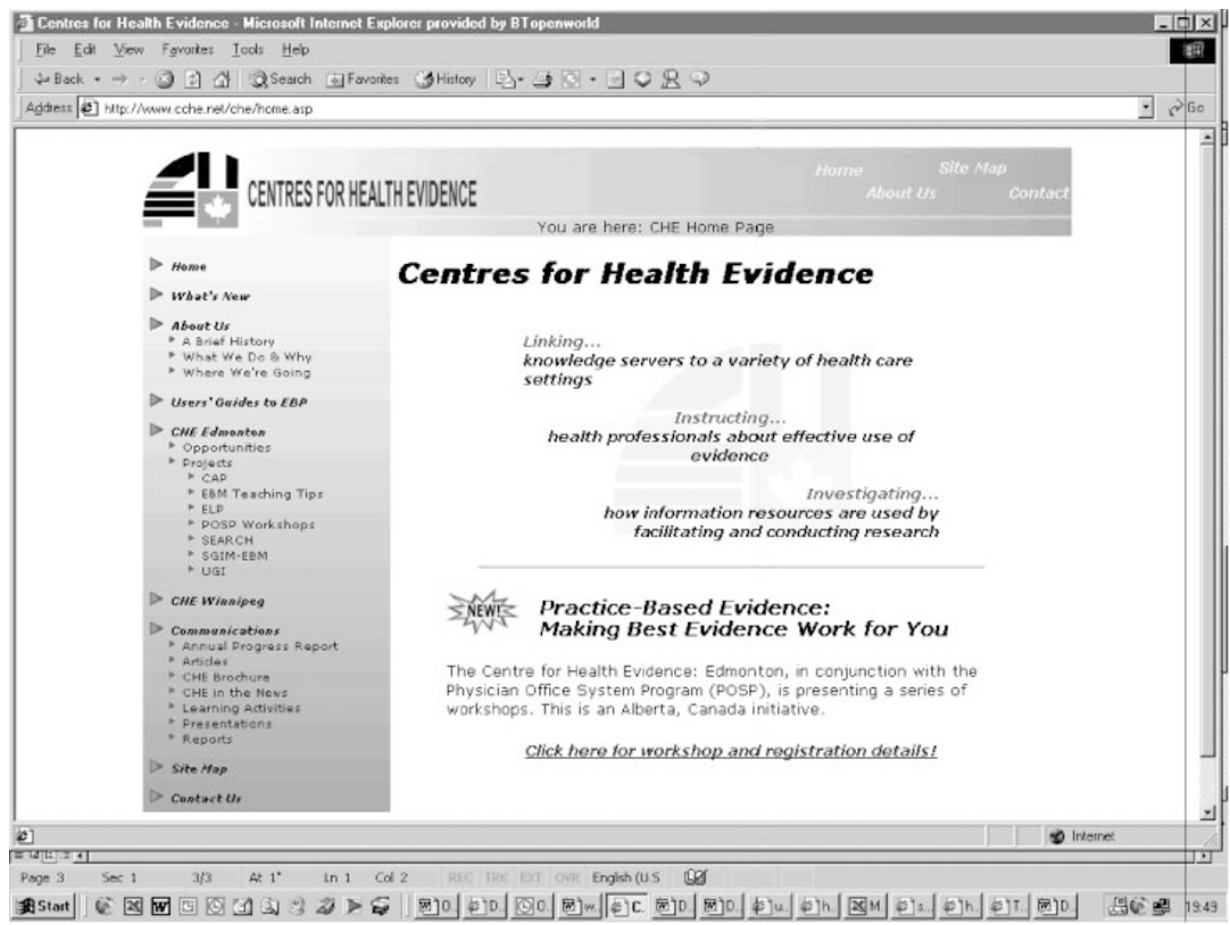

Figure 1 The Centres for Health Evidence-home page

Infostructure objectives:

- identifying, installing, packaging and disseminating health knowledge resources in ways that meet the unique decisionmaking needs of users,

- building and deploying health knowledge servers,

- linking knowledge servers to a variety of health care settings. 
Instruction objectives:

- helping users understand health evidence and the types of knowledge appropriate to different health care problems,

- facilitating on-line training about effective use of best internal and external evidence,

- designing just-in-time learning interventions, experiential teaching methods and continuing professional developmentas-you-work.

\section{Investigation objectives:}

- capturing subjective and objective data about how information resources are used,

- monitoring the effects of information behaviours on health decisions,

- facilitating research on the effects of alternative knowledge packaging and dissemination strategies.

To assist in meeting these objectives the Centres for Health Evidence-website was established in 1999 with funding from the Canadian Office of Health and Alberta Heritage Foundation for Medical Research, the Universities of Alberta and Manitoba, the Capital Health Authority (Edmonton), the Winnipeg Regional Health Authority, InfoWard Inc. now iW Technologies Inc.), and the Multimedia Advanced Computational Infrastructure. The current format of the web site was launched in October 2001 see figure 1.

The overall presentation is clear and uncluttered and it is regularly updated. Navigation of the site is straight forward and it is easy to access the more important features of the site. For the evidence-based practitioner there are two clear high points in this website. The first and probably the best used are the users' guides to evidence-based practice pages. These pages contain the complete set of users' papers as published in the Journal of the American Medical Association (figures 2 and 3 ).

The only disappointing element of these pages is that the tools eg. calculators, worksheets and other educational material are not available. Despite this these pages represent a useful resource for those seeking tools to help them critically appraise the various different study designs found in the literature. However, updates and enhanced versions of these tools can be found at

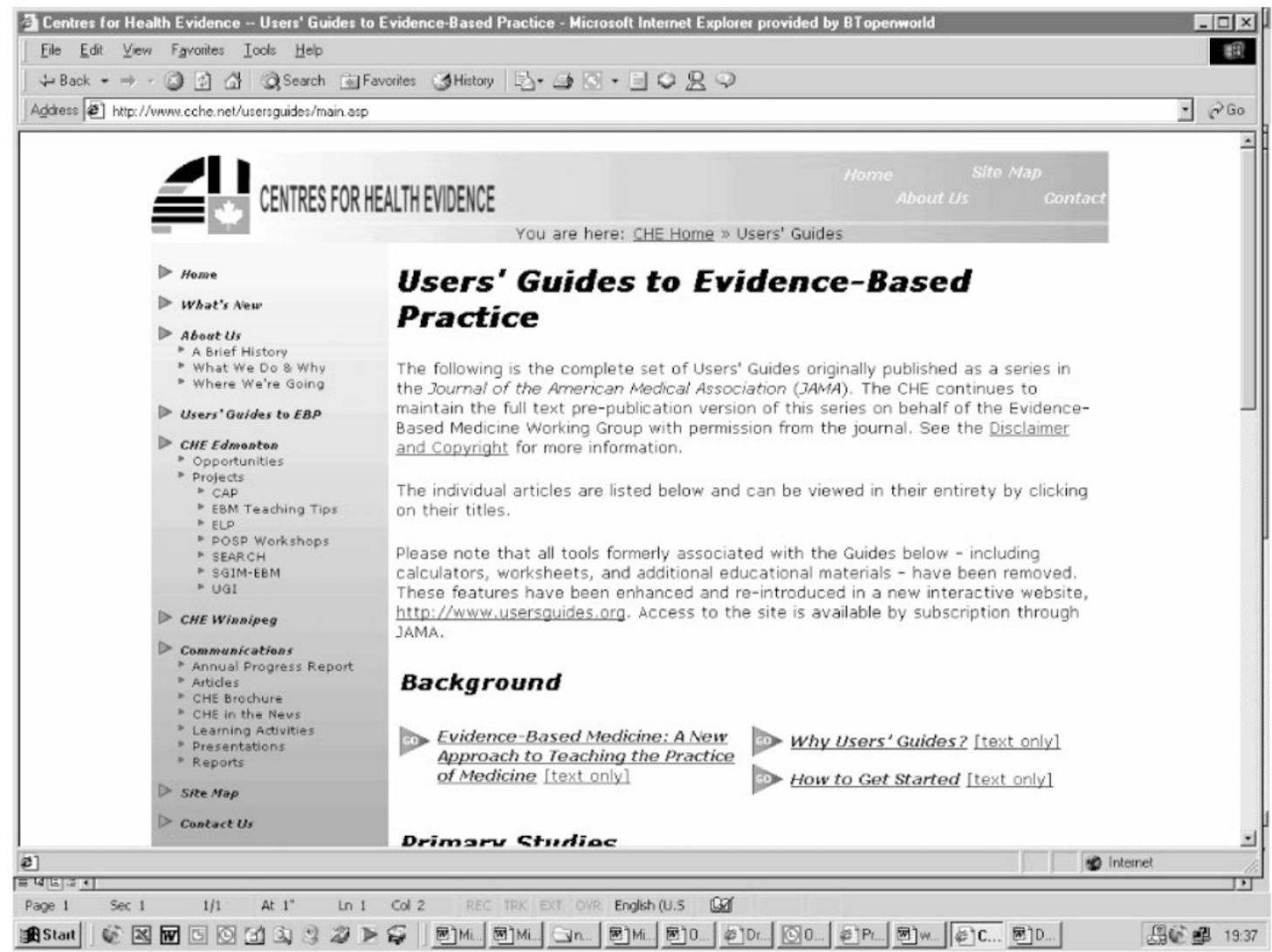

Figure 2 The users guide page 


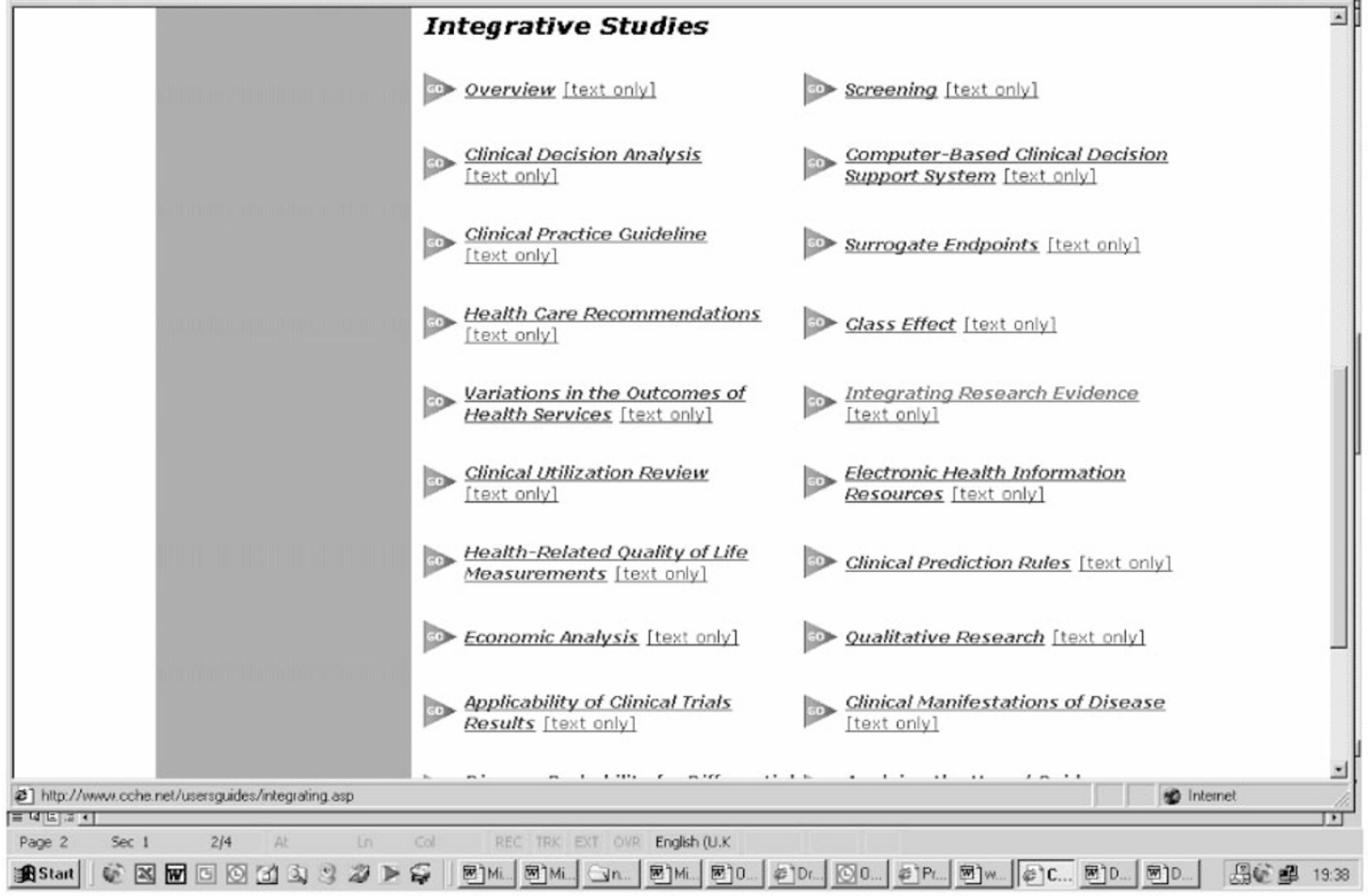

Figure 3 Users' guide page showing part of the list of papers

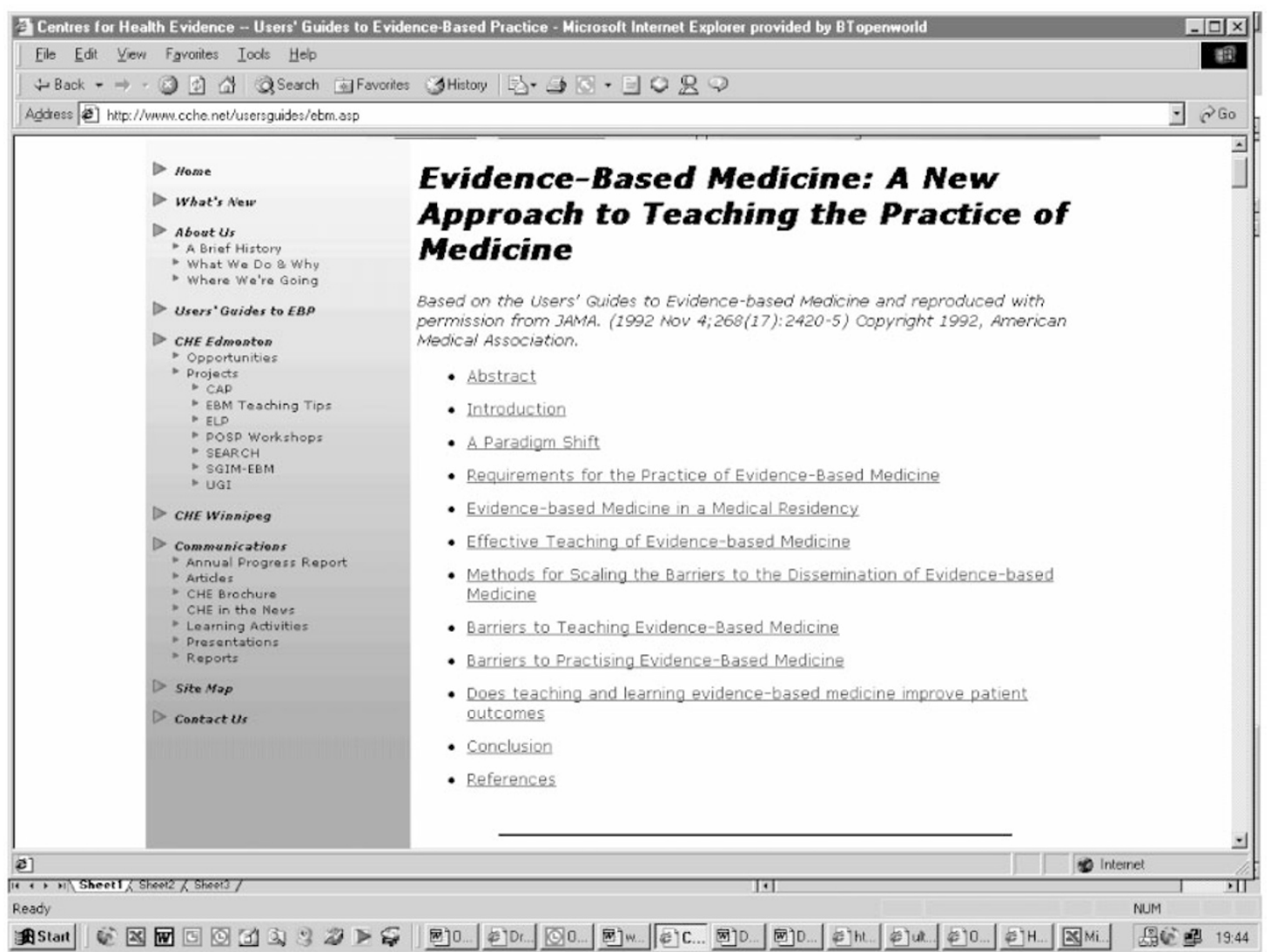

Figure 4 Evidence-based Practice: A new approach to the teaching and practice of medicine page from www.cche.net 
the users' guide website, www.usersguides.org, a subscription site available through the Journal of the American Medical Association.

The other useful section of the website are the pages containing the full text of the book Evidence-based Practice: A new approach to the teaching and practice of medicine (Figure 4). As with the users guides the tools are not available but the text provides a good overview of the evidence-based approach.

Overall this website is a useful resource for evidence-based practitioners and is one I would recommend that you keep it on your favourites list. 